\title{
Iron Deficiency Induced Changes in Iron Reductase Activity in Papaya Roots
}

\author{
Thomas E. Marler, ${ }^{1}$ Ruben dela Cruz, and Andrea L. Blas \\ College of Agriculture and Life Sciences, University of Guam, UOG Station, Mangilao, Guam 96923
}

\begin{abstract}
AdDitional INDEX WORDs. reductase, strategy I iron efficiency
Abstract. Four papaya (Carica papaya L.) cultivars were cultured aeroponically or in perlite to determine the magnitude, timing, and root locality of Fe reductase induced by Fe deficiency. Five soybean [Glycine max (L.) Merrill] lines with a known range of Fe-deficiency chlorosis scores were cultured in perlite for comparison. Speed of inducement of $\mathrm{Fe}$ reductase activity was determined in plants cultured without $\mathrm{Fe}$ for 0 to 17 days. Location of Fe reductase activity was determined by sectioning roots from the tip to 60 to $70 \mathrm{~mm}$ proximal to the root tip from plants cultured without Fe for 16 to 19 days. The Fe reductase system was induced in all papaya cultivars after 7 to 11 days without Fe, and activity increased through 17 days. Iron reductase activity in all papaya cultivars was comparable to the most tolerant soybean line. The zone of highest activity was the apical $10 \mathrm{~mm}$ of roots. These results indicate that papaya roots are highly efficient in induced Fe reductase activity. The highest activity in root tips underscores the importance of maintaining a healthy, continually growing root system with numerous growing points when culturing papaya in alkaline substrates.
\end{abstract}

Iron deficiency induces physiological changes in the roots of some plants that increase Fe uptake from alkaline soils. Uptake of $\mathrm{Fe}$ into the cytoplasm is restricted to $\mathrm{Fe}^{2+}$ for dicots (Marschner, 1995), yet soil reaction maintains $\mathrm{Fe}$ as $\mathrm{Fe}^{3+}$ in alkaline soils. The physiological changes address these soil reaction issues, and are represented by a complex set of responses. Some of these responses include an $\mathrm{Fe}$ reductase system capable of reducing $\mathrm{Fe}^{3+}$ to $\mathrm{Fe}^{2+}$, release of reductants into the rhizosphere, an increase of organic acids with chelating properties, and release of protons which reduces $\mathrm{Fe}^{3+}$ to $\mathrm{Fe}^{2+}$ by directly influencing soil reaction (Landsberg, 1981; Marschner et al., 1986; Olsen and Brown, 1980; Römheld, 1987). For dicots, the inducible changes and resulting increase in Fe acquisition have been characterized as strategy I iron efficiency (Römheld and Marschner, 1986).

Papaya (Carica papaya) is cultivated throughout the equatorial regions, and has naturalized in many tropical lowland habitats. The optimum soil $\mathrm{pH}$ for papaya cultivation reportedly ranges from 5.5 to 7.0 (Awada et al., 1975; Marler et al., 1994). Papaya root growth is limited in acid soils, and correction for Ca deficiency and Al toxicity improves root growth (Marler and dela Cruz, 2001). Growth and morphology of papaya seedlings grown in complete nutrient solution were relatively unaffected within the $\mathrm{pH}$ range of 4.0 to 8.8 (Marler, 1998). Furthermore, Manshardt and Zee (1994) reported that calcareous soils characterize the center of origin for the species. Commercial production of papaya in calcareous soils occurs in southern Florida (Malo and Campbell, 1986) and northern Guam. We have not observed Fe deficiency symptoms on papaya plants growing in Guam, except following root damage by poor aeration or canopy damage by tropical cyclones.

Kannan (1985) reported that acidification of the rooting medium occurred when papaya was grown in Fe-deficient media. No other reports have included the extent of strategy I mechanisms associated with Fe efficiency for this species. Since papaya plants thrive in calcareous soils, we hypothesized that the species may

Received for publication 19 Jan. 2001. Accepted for publication 20 Nov. 2001 We thank Howard Gabe of Novartis Seeds, Inc., Golden Valley, Minn., for donation of soybean seeds and providing field evaluation data on soybean irondeficiency chlorosis. We thank David W. Reed for advice on assay procedures, Rick Wood for use of equipment and aid in setting up lab procedures, and Haluk Discekici for use of equipment.

'Corresponding author; e-mail: tmarler@uog.edu. be endowed with an Fe reductase system induced by Fe deficiency which is a characteristic attribute of strategy I Fe efficiency. Therefore, the objective of this study was to determine the magnitude, timing, and root locality of the Fe reductase system for papaya roots.

\section{Materials and Methods}

Plant material and culture. Four papaya cultivars were used in the study, two from the Solo group ('Sunrise' and 'Waimanalo') and two from the Formosa group ('Tainung 2' and 'Red Lady'). Papaya breeding programs have focused primarily on fruit characters and more recently on disease resistance. Choice of cultivar in production is determined largely by target market in Guam. The Asian and Pacific Islands markets require the larger fruit of the Formosa group, which have been selected for fresh weight generally $>1.5 \mathrm{~kg}$. The remainder of the markets prefer the smaller fruit of the Solo group, which are up to $\approx 0.5 \mathrm{~kg}$ in weight. To our knowledge, there have been no observations of variations among papaya cultivars in terms of soil chemistry or response to calcareous soils. The two cultivars representing each group were selected because they are highly characteristic of their group and are widely planted.

Seeds of the four cultivars were sown on 2 Feb. 1999 in flats with $7.5-\mathrm{cm}$-diameter cells ( $280 \mathrm{~mL}$ capacity) using Sunshine Mix 4 (Sun Gro Hort., Bellevue, Wash.). Germination and growth through 25 Mar. 1999 occurred under a polypropylene covered shelter with $90 \%$ to $92 \%$ sunlight transmission. The plants received a weekly fertilization drench of a complete nutrient solution based on $7.5 \mathrm{~mm}$ nitrogen (Peter's Excel; Teufel Nursery, Inc., Woodinville, Wash.). Mean daily air temperature extremes were $24 \pm 2{ }^{\circ} \mathrm{C}$ and $33 \pm 1{ }^{\circ} \mathrm{C}$.

Plants were divided into two groups on 25 Mar. 1999 for separate experiments conducted underneath the same polypropylene rain shelter. One group was bare-rooted and transferred to aeroponics where roots were misted with half-strength Hoagland's solution ( $\mathrm{pH} \approx 6.5$ ) (Hoagland and Arnon, 1950). The systems were comprised of 150 -L containers containing $\approx 30 \mathrm{~L}$ of solution. Submersible pumps were used to supply suspended mist nozzles with the nutrient solution such that the entire volume of the aeroponics system was continuously being sprayed with the solution. Plants were held in place with foam after inserting the 
roots into holes on the top of the chambers. The plants were arranged in a randomized complete block with eight replications. Iron was supplied in the solution at $5 \mathrm{mg} \cdot \mathrm{L}^{-1}$ as Fe-EDDHA [ethylenediamine di (O-hydroxyphenyl acetic acid)]. The solution was switched to half-strength Hoagland's solution without Fe on 1 Apr. 1999, after running several changes of reverse osmosis water through the aeroponics system for $12 \mathrm{~h}$. Thereafter, the minus-Fe solution was changed on weekly intervals. The reductase assay was conducted on 16, 18, and $19 \mathrm{~d}$ of growth in the minus-Fe solution.

Each plant in the second group was replanted into a $625-\mathrm{mL}$ plastic container filled with washed perlite. Each container was drenched with half-strength Hoagland's solution plus Fe on a daily basis. On 3 Apr. and at 3, 6, 10, and 13 d later, four plants per cultivar were switched to receiving daily drenching of halfstrength Hoagland's solution minus Fe. Four plants per cultivar, designated as control plants, received the complete solution until the day of the assay. Each container was flushed with reverse osmosis water at least 10 times throughout the photoperiod on the day the switch from plus-Fe to minus-Fe solution occurred. The reductase assay was conducted on 20 Apr. 1999 after plants had grown without $\mathrm{Fe}$ for $0,4,7,11,14$, or $17 \mathrm{~d}$. These plants were arranged in a randomized complete block design $(n=4)$. We used this approach to determine the timing of Fe reductase activity because switching individual replications to minus-Fe was not possible in the aeroponics systems.

We also included several soybean (Glycine max) cultivars to provide a frame of reference for the papaya data, since the $\mathrm{Fe}$ reductase activity for soybean has been heavily studied and that for papaya has not been reported previously. Furthermore, Fe reductase activity for soybean is highly correlated with visual scores of Fe-deficiency chlorosis (Jolley et al., 1992). Five soybean breeding lines from Novartis Seeds, Inc. (Golden Valley, Minn.) were used, based on a range of Fe-deficiency chlorosis ratings from multiple years and sites. Based on their data, two soybean lines were tolerant (S66-90 and S83-30), two were intermediate (S57-11 and RA452), and one was sensitive (S7555) (Howard Gabe, personal communication). Seeds were planted directly in washed perlite in 625-mL plastic containers on 1 Apr. 1999, and received the complete nutrient solution drench until 9 Apr. Thereafter, the plants received the minus-Fe solution until $20 \mathrm{Apr}$. when the assay was conducted. The plants were arranged in a randomized complete block design $(n=4)$.

IRON REDUCTASE ASSAY. The entire root systems of plants from the aeroponics system were harvested and rinsed in distilled water. The plants were harvested by block. Four types of root segments were assayed. Primary lateral roots grew $\geq 20 \mathrm{~mm} \cdot \mathrm{d}^{-1}$, and developed short, thin secondary roots (10 to $20 \mathrm{~mm}$ long). One root segment assayed was the terminal $10 \mathrm{~mm}$ of these short secondary roots. The other three segments assayed were based on distance from the tip of the primary lateral roots ( 0 to 10,30 to 40 , and 60 to $70 \mathrm{~mm}$ ). The number of roots included from each plant was 6 to 7 for the 3 segments from the primary lateral roots, and 10 to 12 for the secondary roots. After four blocks were assayed, it was apparent that the Fe reductase activity in the 0 to $10 \mathrm{~mm}$ segment declined to a relatively low level by the 30 to $40 \mathrm{~mm}$ segment. Thus, we modified the methods for the four remaining blocks to characterize more fully the Fe reductase activity near the root tip. The segments prepared for assay from these plants were 0 to 5,5 to 10,10 to 15 , and 15 to $20 \mathrm{~mm}$. The number of roots included from each plant was 10 to 12 .

Papaya and soybean plants cultured in perlite were bare- rooted and the intact root system was rinsed in distilled water before excising 6 to 7 papaya or 10 to 12 soybean primary root tips of $10 \mathrm{~mm}$ length. These plants were harvested by block.

Root sections were rinsed in distilled water before assay using methods similar to Albano and Miller (1996). They were bathed in a $0.2 \mathrm{~mm} \mathrm{CaSO}_{4}$ solution for $10 \mathrm{~min}$ then immersed in $3 \mathrm{~mL}$ oxygenated assay solution containing $0.2 \mathrm{mM} \mathrm{CaSO}_{4}, 0.1 \mathrm{~mm}$ $\mathrm{Fe}^{3+}$ EDTA (ethylenediamine-tetraacetic acid), and $0.3 \mathrm{~mm}$ of the ferrous chelator BPDS (4,7-diphenyl-1,10-phenanthrolinedisulfonic acid). The solution was buffered at $\mathrm{pH} 5.5$ with $5 \mathrm{~mm}$ MES [2-(N-morpholino) ethanesulfonic acid]. Assays were conducted in the dark at $23{ }^{\circ} \mathrm{C}$. The appearance of $\mathrm{Fe}^{2+}$-BPDS in the assay solution was determined at $120 \mathrm{~min}$ by absorbance at 535 nm (DR-200; Hach, Loveland, Colo.). Blanks (assay solution minus roots) were assayed at least every hour to quantify autoreduction (background Fe reduction of the assay conditions). The fresh weight (FW) of the roots in each sample was determined at the end of the incubation period. The assays were conducted between 0900 and $1800 \mathrm{HR}$, and the assays for each block were completed in $\leq 2 \mathrm{~h}$.

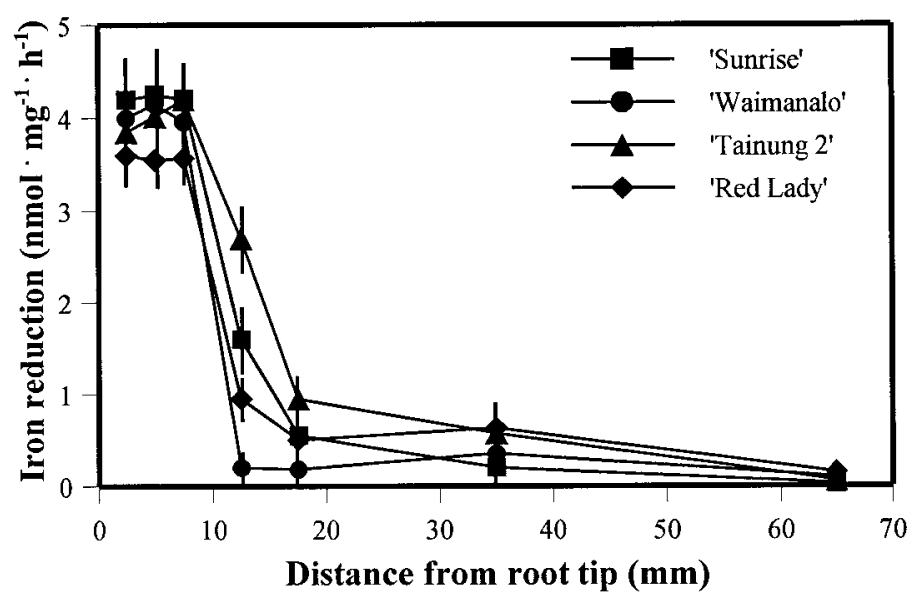

Fig. 1. Iron reduction of excised root segments of four papaya cultivars grown for 16 to $19 \mathrm{~d}$ without $\mathrm{Fe}$ in aeroponics culture as a function of distance from the root tip. Vertical bars indicate SE $(n=4)$.

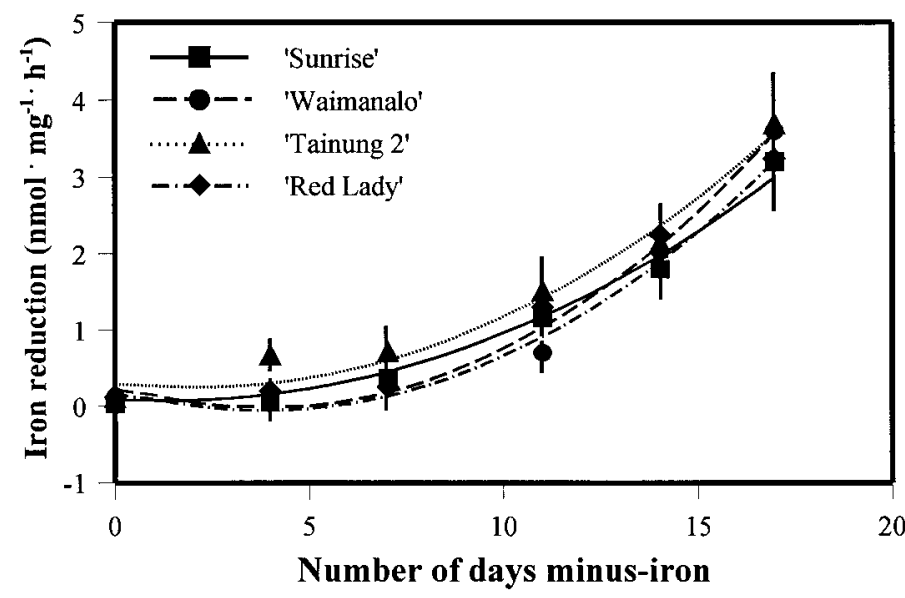

Fig. 2. Iron reduction of excised root tips $(10 \mathrm{~mm})$ from four papaya cultivars growing in perlite as a function of days without Fe supplied to the roots. Regression lines: for 'Red Lady', Fe reduction $=0.221-0.144 \times d+0.0188 \times$ $\mathrm{d}^{2}, r^{2}=0.90$; for 'Sunrise', Fe reduction $=0.101-0.034 \times \mathrm{d}+0.0120 \times \mathrm{d}^{2}, r^{2}=$ 0.88 ; for 'Tainung 2', Fe reduction $=0.317-0.064 \times \mathrm{d}+0.0150 \times \mathrm{d}^{2}, r^{2}=0.93$; and for 'Waimanalo', Fe reduction $=0.291-0.161 \times \mathrm{d}+0.0208 \times \mathrm{d}^{2}, r^{2}=0.98$. Vertical bars indicate SE $(n=4)$. 
Calculations and statistics. The concentration of $\mathrm{Fe}^{2+}-$ BPDS produced was calculated using an extinction coefficient of 22.14/mm per cm (Welch et al., 1993). Autoreduction was minimal, but was included in calculations by averaging two sequential blanks and subtracting this mean from each root assay which fell between the two blanks. Total reduction per $3 \mathrm{~mL}$ of solution was standardized to a unit root FW included in each assay. Total root FW ranged from 8 to $20 \mathrm{mg}$ for short, secondary papaya roots, 15 to $60 \mathrm{mg}$ for primary papaya roots, and 20 to $50 \mathrm{mg}$ for soybean roots.

The pattern of $\mathrm{Fe}$ reduction as a function of culture time without $\mathrm{Fe}$ was determined using regression analysis, with the number of days defined as the independent variable. The resulting curves for the four cultivars were represented by quadratic equations. Equations were tested by analysis of covariance for homogeneity among the cultivars. Papaya root $\mathrm{Fe}$ reduction data are expressed as a function of root locality and soybean root $\mathrm{Fe}$ reduction data are presented as means of four replications \pm SE.

\section{Results}

We did not observe any canopy symptoms indicative of $\mathrm{Fe}$ deficiency in any of the papaya plants. Iron reduction in the terminal $5 \mathrm{~mm}$ of papaya lateral roots in the aeroponics study was 3.5 to $4.2 \mathrm{nmol} \cdot \mathrm{mg}^{-1} \cdot \mathrm{h}^{-1}$, and no differences occurred among the four cultivars (Fig. 1). Iron reduction remained high in the 5 to 10 $\mathrm{mm}$ section, but declined by $\approx 5 \%$ for 'Waimanalo' to $\approx 65 \%$ for 'Tainung 2' at 10 to $15 \mathrm{~mm}$ from the root apex. Iron reduction potential declined more in the 15 to $20 \mathrm{~mm}$ section, ranging from $5 \%$ to $25 \%$ of the 0 to $5 \mathrm{~mm}$ values. Iron reduction was negligible at 60 to $70 \mathrm{~mm}$ from the root terminal.

Iron reduction potential in the short secondary root tips was slightly lower than in primary root tips (data not presented), and ranged from $2.3 \pm 0.5 \mathrm{nmol} \cdot \mathrm{mg}^{-1} \cdot \mathrm{h}^{-1}$ for 'Red Lady' to $4.3 \pm 0.6$ $\mathrm{nmol} \cdot \mathrm{mg}^{-1} \cdot \mathrm{h}^{-1}$ for 'Sunrise'. Values exceeded Fe reduction levels found in primary lateral roots beyond $10 \mathrm{~mm}$ from the tip.

Fig. 3. Iron reduction of excised root tips $(10 \mathrm{~mm})$ from five soybean cultivars with a range of Fe-deficiency chlorosis when grown in alkaline soils. Cultivars were grown in perlite for $11 \mathrm{~d}$ without Fe supplied to the roots. Vertical bars indicate SE $(n=4)$.

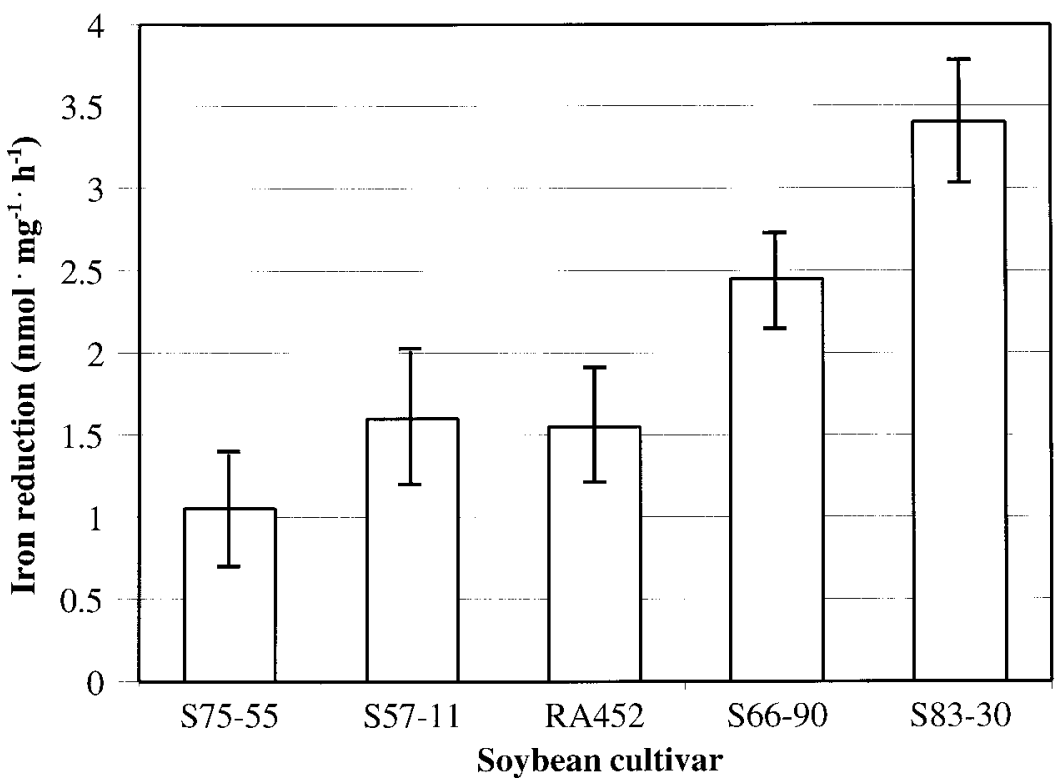

Development for induced reductase activity as a function of time fit quadratic equations (Fig. 2). Analysis of covariance test of homogeneity of the equations indicated the four equations could not be used to describe the same response curve $(P \leq 0.029)$. Thus, the timing of initial inducement of the reductase system differed among the cultivars.

Iron reduction increased in 'Tainung 2' roots $4 \mathrm{~d}$ after culture without $\mathrm{Fe}$, and increased in roots of all four cultivars by day 11 (Fig. 2). Iron reduction in 'Waimanalo' roots increased more slowly than in the other three cultivars, but by day 14 , the four cultivars were similar.

Iron reduction for the soybean cultivars followed the same pattern as the visual $\mathrm{Fe}$-chlorosis scores from previous field trials. The cultivar with the worst chlorosis scores (S75-55) exhibited a reduction potential of only $1 \mathrm{nmol} \cdot \mathrm{mg}^{-1} \cdot \mathrm{h}^{-1}$, the two with the best chlorosis scores (S66-90 and S83-30) exhibited the highest reduction potential, and the two with the intermediate chlorosis scores (S57-11 and RA 452) exhibited intermediate reduction potential (Fig. 3). Iron deficiency chlorosis was apparent in the S75-55, S57-11, and RA 452 plants by day 5 of induction.

\section{Discussion}

Papaya roots responded to Fe deficiency with enhanced $\mathrm{Fe}$ reduction. Induction was slower than in efficient soybean cultivars (Jolley et al., 1992), but the magnitude under the conditions of this study was comparable with that of the efficient soybean line S83-30 and greater than the other four soybean lines (Fig. 3). If these four cultivars are representative of the species, papaya roots are highly efficient at $\mathrm{Fe}$ reduction. There were no obvious differences between the Formosa cultivars and the Solo cultivars. Thus, both breeding programs appear to have retained root reductase potential throughout their selection processes.

The short lateral roots were highly active in Fe reduction. These short thin roots are ephemeral, typically lasting 1 to 2 weeks on plants growing in mineral soil (T. Marler, unpublished data). Our results indicate that these ephemeral roots play an important role in Fe nutrition during their short life span, as they allow any given length of pioneer root to mine a greater volume of soil during the temporary increase in number of root tips.

Induced Fe reduction segregated among the cultivars in the first few days after induction but converged by day 14 (Fig. 2). The small differences among the cultivars are probably of little consequence in the field. A healthy papaya plant is supported by the continual appearance of new root tips. Thus, the presence of fully induced root tips should be plentiful as long as there is no arrest in root system development.

The importance of maintaining a healthy, continually expanding root system is underscored by this study. Iron reductase activity in the 10 to $15-\mathrm{mm}$ root section was greatly reduced in all four cultivars when compared with the root tips, and was negligible in the 60 to $70 \mathrm{~mm}$ root section (Fig. 1). Since these roots grew at a rate of $\geq 20$ $\mathrm{mm} \cdot \mathrm{d}^{-1}$, the duration of the site of highest $\mathrm{Fe}$ reductase activity for any given segment along the axis of the growing root was $<24 \mathrm{~h}$. Thus, any decline in root extension rate or the total number of root tips may strongly influence total $\mathrm{Fe}$ acquisition potential. These implications are even more important when considering this inducible reductase has shown activity for other metal cations (Welch et al., 1993). 
The slightly higher Fe reductase activity observed on root tips of the aeroponics plants (Fig. 1) compared with the perlite plants (Fig. 2) may have been due to our procedures. We did not use any method of Fe scavenging from root or hardware surfaces when the culture solution was switched from plus-Fe to minus-Fe. Thus, the amount of trace $\mathrm{Fe}$ residue in the aeroponics system was probably less than in the perlite system because of greater surface area with the perlite substrate. As a result, Fe reduction potential may have developed more quickly in the aeroponics system, and may account for the slightly greater values from this culture system.

We have observed increased Fe-deficiency chlorosis of Solo and Formosa papaya plants growing in calcareous soils following foliage damage by tropical cyclones on Guam. A common response to canopy defoliation or pruning is a decline in root growth until the canopy recovers (Eissenstat and Duncan, 1992). Using observation windows, we have observed that defoliation leads to a rapid decline in root extension and the total number of root tips of papaya plants (Marler and Stushnoff, 1999). Results of the present study indicate that this decline in root extension following canopy damage is likely the cause of increased $\mathrm{Fe}$ deficiency symptoms, since reduction potential is primarily in the growing root tips.

\section{Literature Cited}

Albano, J.P. and W.B. Miller. 1996. Iron deficiency stress influences physiology of iron acquisition in marigold (Tagetes erecta L.). J. Amer. Soc. Hort. Sci. 121:438-441.

Awada M., R. Suehisa, and Y. Kanehiro. 1975. Effects of lime and phosphorus on yield, growth, and petiole composition of papaya. J. Amer. Soc. Hort. Sci. 100:294-298.

Eissenstat D.M. and L.W. Duncan. 1992. Root growth and carbohydrate responses in bearing citrus trees following partial canopy removal. Tree Physiol. 10:245-257.

Hoagland D.R. and D.I. Arnon. 1950. The water culture method for growing plants without soil. Calif. Agr. Expt. Sta. Circ. 347.

Jolley V.D., D.J. Fairbanks, W.B. Stevens, R.E. Terry, and J.H. Orf. 1992. Root iron-reduction capacity for genotypic evaluation of iron efficiency in soybean. J. Plant Nutr. 15:1679-1690.

Kannan, S. 1985. Fe-deficiency tolerance in papaya (Carica papaya L.): $\mathrm{pH}$ reduction and chlorosis recovery in response to stress. J. Plant Nutr. 8:1191-1197.

Landsberg, E.C. 1981. Organic acid synthesis and release of hydrogen ions in response to Fe deficiency stress of mono- and dicotyledonous plant species. J. Plant Nutr. 3:579-591.

Malo, S.E. and C.W. Campbell. 1986. The papaya. Univ. of Fla., Gainesville, Coop. Ext. Serv. Fruit Crops Fact Sheet FC-11.

Manshardt, R.M. and F.T.P.Zee. 1994. Papaya germplasm and breeding in Hawaii. Fruit Var. J. 48:146-152.

Marler T.E. 1998. Solution pH influences on growth and mineral element concentrations of 'Waimanalo' papaya seedlings. J. Plant Nutr. 21:26012612.

Marler, T.E. and R. dela Cruz. 2001. Chemical factors enhancing papaya root growth in a tropical volcanic acid subsoil. HortScience 36:10371038.

Marler, T.E., A.P. George, R.J. Nissen, and P.C. Andersen. 1994. Miscellaneous tropical fruits, p. 199-224. In: B. Schaffer and P.C. Andersen (eds.). Handbook of environmental physiology of fruit crops. vol. 2. Subtropical and tropical crops. CRC Press, Boca Raton, Fla.

Marler, T.E. and C. Stushnoff. 1999. Root, stem, and fruit growth of 'Tainung 1' papaya plants following defoliation. HortScience 34:488489 (abstr.).

Marschner, H. 1995. Mineral nutrition of higher plants. $2^{\text {nd }}$ ed. Academic Press, London.

Marschner, H., V. Römheld, and M. Kissel. 1986. Different strategies in higher plants in mobilization and uptake of iron. J. Plant Nutr. 9:695713.

Olsen, R.A. and J.C. Brown. 1980. Factors related to iron uptake by dicotyledonous and monocotyledonous plants: I. pH and reductant. J. Plant Nutr. 2:629-645.

Römheld, V. 1987. Different strategies for iron acquisition in higher plants. Physiol. Plant. 70:231-234.

Römheld, V. and H. Marschner. 1986. Mobilization of iron in the rhizosphere of different plant species. Adv. Plant Nutr. 2:155-204.

Welch, R.M., W.A. Norvell, S.C. Schaefer, J.E. Shaff, and L.V. Kochian. 1993. Induction of iron (III) reduction in pea (Pisum sativum L.) roots by $\mathrm{Fe}$ and $\mathrm{Cu}$ status: Does the root-cell plasmalemma Fe(III)-chelate reductase perform a general role in regulating cation uptake? Planta 190:555-561. 